\title{
Modelling the Non-radiative Singlet Excited State Isomerization of Diphenyl-Acetylene: A Vibronic Coupling Model.
}

\author{
Christopher Robertson ${ }^{\mathrm{a}, \mathrm{b}}$, G. A. Worth ${ }^{\mathrm{a}, \mathrm{c}}$ \\ ${ }^{a}$ School of Chemistry, University of Birmingham, Birmingham, B15 2TT, U.K. \\ ${ }^{b}$ Present address: Dept. of Chemistry, University of Warwick, Gibbet Hill, Coventry, \\ CV4 7AL, U.K. \\ ${ }^{c}$ Present Address: Dept. of Chemistry, University College London, 20, Gordon St., \\ WC1H OAJ, U.K.
}

\begin{abstract}
Tolane (diphenyl-acetylene) is the smallest component of macromolecular arrays known as dendrimers that have interesting energy transport properties after photo-excitation. In this paper, a vibronic coupling Hamiltonian is set up to describe the initial internal conversion of this molecule. The calculated absorption spectrum is in good agreement with experiment, with the ordering of states and energies from MRCI-DFT calculation. The focus of the study is the pathway for photo-excited isomerisation from the linear geometry at the Frank-Condon point to a trans- structure. The model shows that the origin of the excited-state minimum for the trans-isomer is due to stabilisation of a high lying state. Quantum dynamics calculations using the MCTDH algorithm show the model agrees with experiment that isomerisation only occurs at high temperature. It also suggests that internal conversion to the $\mathrm{S}_{1}$ global minima happens via second order coupling terms, which can explain the observed picosecond timescales.
\end{abstract}

Keywords: Tolane; Diphenyl-acetylene; Dendrimer Photochemistry; Vibronic Coupling Model; MCTDH

\footnotetext{
Email addresses: c.robertson@warwick.ac.uk (Christopher Robertson), g.a.worth@ucl.ac.uk (G. A. Worth)

URL: http://chemb125.ucl.ac.uk/worthgrp/ (G. A. Worth)
} 


\section{Introduction}

Diphenyl-acetylene (known as tolane or DPA) is the monomial of a family of extended dendrimer segments exhibiting remarkable photo-funnelling properties [1]. These dendrimers show much promise in applications such as photoactive arrays in polymeric systems [2] and single-molecular light emitting diodes (LEDs) [3]. Tolane has been studied more than any other dendrimer segment $[4,5,6,7,8,9,10,11,12,13]$, and an overall mechanism of its photo-relaxation is emerging, but not yet complete.

Tolane has been observed experimentally to posses a planar structure of $\mathrm{D}_{2 h}$ symmetry at the ground-state equilibrium geometry [8] (see Fig 1). Hirata et al [6] performed time-resolved experiments and proposed a transfer mechanism $\mathrm{S}_{0} \rightarrow X \rightarrow Y \rightarrow \mathrm{T}_{1}$ with a short-lived (8 ps, or 6.3 ps [14]) optically active state $X$ at $290 \mathrm{~nm}$ (fluorescing at $500 \mathrm{~nm}$ ), being a precursor to a long-lived (200 ps) 'dark' (optically forbidden, fluoresces at $700 \mathrm{~nm}$ ) state $\mathrm{Y}$, which undergoes intersystem crossing to a phosphorescing triplet state $\mathrm{T}_{1}(\sim 1 \mu \mathrm{s})$. The decay of $\mathrm{X}$ was found to coincide directly with the rise time of $\mathrm{Y}$. A temperature dependence for the lifetime of $\mathrm{X}$ indicates a small activation barrier of $0.11 \mathrm{eV}(0.155 \mathrm{eV}[7])$.

The bright state $\mathrm{X}\left(1 \mathrm{~B}_{1 u}\right)$ lies at $4.27 \mathrm{eV}[6]$ or $4.3 \mathrm{eV}[10,9]$ and the dark state $\mathrm{Y}\left(1 \mathrm{~A}_{u}\right)$ at $4.37 \mathrm{eV}$. The latter state has a trans-stilbene geometry at its energy minimum. However, the precise order of states in the $\mathrm{FC}$ region is not clear, with two states of $\mathrm{B}_{2 u}$ and $\mathrm{B}_{3 g}$ symmetries $(4.33 \mathrm{eV}$ ) possibly lying below the bright state in solution [10]. An $\mathrm{A}_{g}$ state has also been posited as the first excited state $[5,9]$.

Saltiel et al [10] suggested that at room temperature there is significant competition between vibrational cooling in the $\mathrm{B}_{1 u}$ state and conversion to the $1 \mathrm{~A}_{u}$, trans-stilbene geometry, and the latter is shut down at $77 \mathrm{~K}$. They also inferred the presence of intersystem crossing, but this time via a higher lying $\mathrm{B}_{2 u}$ state. This transition is, however, orbitally forbidden and is expected to be weak [15]. It was also concluded that the medium plays an important role in determining the relative positions of $\mathrm{B}_{2 u} / \mathrm{B}_{1 u}$, as well as the barrier height to the trans-stilbene $\left(\mathrm{A}_{u}\right)$ state.

Electronic structure calculations have supported the experimental findings, but have been unable to unambiguously determine the order of the first three excited states. Energies, symmetries and ordering of the calculated states found in the literature are provided in Table 1. Ferrante et al [15] suggested that the $2 \mathrm{~A}_{u}$ state crosses $1 \mathrm{~B}_{1 u}$ to become the first excited state 
stable geometry, with a barrier of $0.145 \mathrm{eV}$. Zgierki et al [16] concluded that the crossing between the $\mathrm{A}_{u}$ state and the $\mathrm{B}_{1 u}$ states might not simply occur purely along the central CC stretching coordinate. They place the transstilbene isomerisation barrier at around $0.29-0.4 \mathrm{eV}$, significantly above the experimentally observed value.

Amatatsu et al [17] found the $\mathrm{B}_{3 g}$ and $\mathrm{B}_{2 u}$ states to lie lower than the $\mathrm{B}_{1 u}$ state, which was considered a possibility by other studies $[15,7,10]$. They also noted that $1 \mathrm{~B}_{1 u}$ is the only strong optically active state in this energy region. Although the bending $\mathrm{CC}-\mathrm{C}$ linkage angle is found to change along the reaction coordinate, it was not found to lead directly to the conical intersection (CI) (and the transition pathway). Instead, the molecule needs to drastically extend both the central and linkage bonds (bonds in the aromatic ring do not change significantly). By looking at different conformation pathways that break the $\mathrm{C}_{2 h}$ symmetry, they concluded that the molecule conserves its planar structure throughout the relaxation. Most recently Kramer et al [18] used $\mathrm{ADC}(2)$ calculations and concluded that the $\mathrm{B}_{1 u}$ state is the lowest in energy (although they label it as $\mathrm{B}_{2}$ )) and that while it is the brightest state, its oscillator strength vanishes when the benzene rings have a twisted $90^{\circ}$ configuration. They note that since the $\mathrm{B}_{1 u}$ minima corresponds to a quinoidal structure, tolane tends to remain planar in this state. Recent TD-DFT calculations [13] also make $1 \mathrm{~B}_{1 u}$ the $\mathrm{S}_{1}$ state $(4.48 \mathrm{eV})$, They caution against the direct comparison of vertical excitation energies to experimental absorption spectral peaks. Instead, they show that by calculating a vibronic spectra the $0-0$ transition gives very good agreement with the experiment. They conclude that the spectra is built primarily from four totally symmetric modes; the acetylene bridge stretching modes and the triangular bending and quinoidal stretching modes of the benzene rings.

A standard approach to study problems involving excited-state dynamics and relaxation is to construct a vibronic coupling model [19]: a simple polynomial expansion of diabatic potential energy surfaces and couplings. In this paper, a preliminary vibronic coupling model of the singlet-state manifold of tolane is constructed and used in quantum dynamic simulations to simulate the isomerization after photo-excitation. The expansion coefficients of the diabatic model are chosen so that the eigenvalues of the potential operator map on to the adiabatic potential surfaces. Such models have been shown to accurately reproduce the short-time dynamics of non-adiabatic systems [20, 21, 22, 23, 24]. For describing tolane, normal mode coordinates were used and the diabatic potential energy surfaces expanded around the Frank- 


\begin{tabular}{|c|c|c|c|c|c|}
\hline state & $(\mathrm{a})$ & $(\mathrm{b})$ & $(\mathrm{c})$ & $(\mathrm{d})$ & $(\mathrm{e})$ \\
\hline $\mathrm{S}_{1}$ & $\mathrm{~B}_{1 u}(4.32)$ & $\mathrm{B}_{1 u}(4.13)$ & $\mathrm{B}_{1 u}(4.18)$ & $\mathrm{B}_{2 u}(4.05)$ & $\mathrm{B}_{2}(4.77)$ \\
\hline $\mathrm{S}_{2}$ & $\mathrm{~A}_{u}(4.35)$ & $\mathrm{B}_{2 u}(4.29)$ & $\mathrm{B}_{2 u}(\sim 4.75)$ & $\mathrm{B}_{3 g}(4.05)$ & $\mathrm{B}_{3}(4.90)$ \\
\hline $\mathrm{S}_{3}$ & $\mathrm{~B}_{2 u}(4.83)$ & $\mathrm{B}_{3 g}(4.29)$ & $\mathrm{A}_{u}(\sim 4.75)$ & $\mathrm{B}_{1 u}(4.51)$ & $\mathrm{B}_{1}(4.90)$ \\
\hline $\mathrm{S}_{4}$ & $\mathrm{~B}_{3 g}(4.83)$ & $\mathrm{A}_{u}(4.31)$ & $\mathrm{B}_{3 g}(\sim 4.75)$ & $\mathrm{A}_{u}(4.85)$ & $\mathrm{A}(5.62)$ \\
\hline $\mathrm{S}_{5}$ & $\mathrm{~A}_{u}(5.23)$ & $\mathrm{A}_{g}(4.36)$ & $\mathrm{A}_{g}(\sim 5.30)$ & & $\mathrm{A}(6.33)$ \\
\hline
\end{tabular}

Table 1: Vertical excitation energies of tolane from the literature (eV). Ho et al [13] place the $\mathrm{S}_{1}$ at $4.48 \mathrm{eV}\left(\mathrm{B}_{1 u}\right)$. (a): Gutmann (AM1), [7], (b): Ferrante (INDO/S) [15], (c): Zgierski (B3LYP/6-31G*), [16], (d): Amatatsu (CAS/MP2/D95V+) [17], (e): Kramer $\left(\mathrm{ADC}(2) / \mathrm{cc}-\mathrm{pVDZ}, \mathrm{D}_{2}\right.$ symmetry) [18]

Condon point. Symmetry arguments then provide selection rules determining which expansion coefficients describing the coupling between states and normal coordinates are allowed into the model.

Using quantum dynamics simulations with the multi-configurational timedependent Hartree (MCTDH) method, from a vertical excitation to the bright state an isomerisation to a trans-stilbene geometry in a picosecond timescale is seen, in accordance with previous work. The MCTDH method, developed by Meyer, Manthe and Cederbaum in the early 90s [25, 26, 27] is a particularly powerful algorithm for propagating multi-dimensional wavepackets and still the only method available for the complete study of systems larger than a few atoms. By analysing the symmetries of states and modes involved, all couplings between the relevant electronic states to first and second order were included, which should provide the major pathways of the photo-relaxation process investigated. By including or excluding modes, we were able to shed light on the mechanism and timescales involved, which are in qualitative agreement with experiments.

The model allows us to conclude that, although a conical intersection is present in the plane of the molecule [17], the molecule must undergo out of plane, trans vibrations along the acetylene bridge that enable second order coupling to populate the trans-stilbene, $\mathrm{S}_{1}$ minima. By calculating the absorption spectra of the model we are able to approximately assign the origin of some bands in the experimental spectra. The calculated spectra also led us to assign the relative position of the first three excited states and their contribution to the spectra at low and high temperatures. 


\section{Model construction}

\subsection{Electronic structure and geometry optimisations}

The first step in describing the photo-excited dynamics of a molecule is the examination of the electronic structure of the excited-states available. Table 2 compares the first five excited states obtained with complete active space (CAS) SCF calculations using different active spaces involving the $\pi$ and $\pi^{*}$ MO's built from conjugating p-orbitals. The CAS $(10,10)$ active space is the same as that used by Amatatsu et al[17]. Consequently, we shall make reference to the orbitals provided in Ref [17], though it should be noted that we find the HOMO- 4 in-plane acetylene $\pi$ bond to be of $\mathrm{B}_{2 g}$ and not $\mathrm{B}_{2 u}$ symmetry. The CAS $(12,12)$ includes the next HOMO-5/LUMO+5 and the CAS $(16,16)$ includes all the $\pi \pi^{*}$ orbitals. Calculations were made with Molpro 09 [28].

The CAS calculations situate the states in the same order as Amatatsu et $a l$, and so disagree with the $\operatorname{ADC}(2)$ calculations of Kramer et al[18]. State $\mathrm{B}_{3 g}$ seems to consistently have similar energies as $\mathrm{B}_{2 u}$, with $\mathrm{B}_{1 u}$ lying not far away. Perturbation theory corrections (columns $g$ and $h$ in Table 2) lower the energy of states, but it does not affect the ordering. It is worth noting that augmented basis functions were also tried, but made little difference and did not affect the ordering of the states.

The characterisation of $1 \mathrm{~B}_{2 u}$ and $1 \mathrm{~B}_{3 g}$ is consistent with Amatatsu, primarily involving $\pi-\pi^{*}$ excitations from the HOMO-1 to LUMO or LUMO-1 to HOMO respectively. The $1 \mathrm{~B}_{2 u}$ state has a very weak transition dipole of $0.05 \mathrm{au}$ (in agreement with Ferrante [15]). The bright $1 \mathrm{~B}_{1 u}$ state has a transition dipole of 2.84 au along the vector corresponding to its lowest moment of inertia. It is described primarily by the HOMO-LUMO, out-ofplane $\pi-\pi^{*}$ excitation of the acetylene bridge, but with some contribution from the HOMO-3, an out-of-plane acetylene/benzene hybrid $\pi$ orbital to the $\mathrm{LUMO}+4$ in-plane acetylene $\pi^{*}$ orbitals. Finally, the $2 \mathrm{~A}_{u}$ state responsible for the trans-stilbene geometry is characterized by the HOMO to $\mathrm{LUMO}+4$, the acetylene out-of-plane $\pi$ orbital to the in-plane $\pi^{*}$ orbital.

Since it is experimentally suggested[9] that the first bright state is the $1 \mathrm{~B}_{1 u}$ state, which agrees with the CASSCF calculations, we performed a geometry optimisation in this state to obtain the $1 \mathrm{~B}_{1 u}$ minima, using a CAS $(10,10)$ wavefunction and no symmetry. The same active space was used to locate the $\mathrm{S}_{1}$, trans-stilbene minima (a "ts" prefix label will be used henceforth to refer to this geometry). Bond lengths of the acetylene bridge, 


\begin{tabular}{|c|c|c|c|c|}
\hline State & $(\mathrm{f})$ & $(\mathrm{g})$ & $(\mathrm{h})$ & $(\mathrm{i})$ \\
\hline $\mathrm{S}_{0}$ & -539.31 & -536.05 & -537.74 & -536.17 \\
\hline $\mathrm{S}_{1}$ & $4.11\left(\mathrm{~B}_{1 u}\right)$ & $4.68\left(\mathrm{~B}_{2 u}\right)$ & $4.71\left(\mathrm{~B}_{2 u}\right)$ & $4.88\left(\mathrm{~B}_{2 u}\right)$ \\
\hline $\mathrm{S}_{2}$ & $4.15\left(\mathrm{~B}_{2 u}\right)$ & $4.68\left(\mathrm{~B}_{3 g}\right)$ & $4.72\left(\mathrm{~B}_{3 g}\right)$ & $4.88\left(\mathrm{~B}_{3 g}\right)$ \\
\hline $\mathrm{S}_{3}$ & $4.16\left(\mathrm{~B}_{3 g}\right)$ & $4.88\left(\mathrm{~B}_{1 u}\right)$ & $5.04\left(\mathrm{~B}_{1 u}\right)$ & $6.53\left(\mathrm{~B}_{1 u}\right)$ \\
\hline $\mathrm{S}_{4}$ & $4.81\left(\mathrm{~A}_{u}\right)$ & $5.74\left(\mathrm{~A}_{u}\right)$ & $6.05\left(\mathrm{~A}_{u}\right)$ & $6.59\left(\mathrm{~A}_{g}\right)$ \\
\hline $\mathrm{S}_{5}$ & $5.04\left(\mathrm{~A}_{g}\right)$ & $6.10\left(\mathrm{~A}_{g}\right)$ & $6.21\left(\mathrm{~A}_{g}\right)$ & $6.96\left(\mathrm{~A}_{u}\right)$ \\
\hline $2 \mathrm{~A}_{u}$ & $5.56\left(\mathrm{~S}_{9}\right)$ & $6.55\left(\mathrm{~S}_{9}\right)$ & $7.12\left(\mathrm{~S}_{9}\right)$ & $7.96\left(\mathrm{~S}_{9}\right)$ \\
\hline
\end{tabular}

Table 2: Energies of the the excited states of tolane. Absolute energy of $\mathrm{S}_{0}$ given in Hartree and all other states energies are relative to that $(\mathrm{eV})$. Bottom row indicates which adiabatic state the $2 \mathrm{~A}_{u}$ is at the ground state minima (energy provided). f: DFTMRCI/TZVP, g: SA-CAS(10,10)+RS2C/cc-pVDZ, h: SA-CAS(12,12)+RS2C/cc-pVDZ, i: $\operatorname{SA}-\mathrm{CAS}(16,16) / \mathrm{cc}-\mathrm{pVDZ}$.

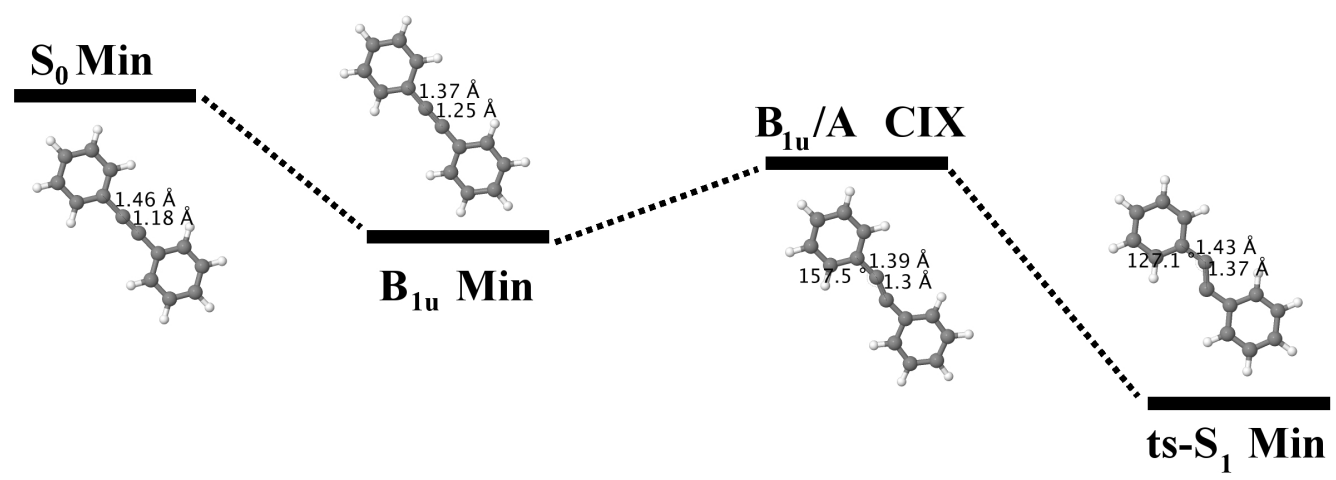

Figure 1: Sequence of geometries joining the $\mathrm{B}_{1 u}$ bright state at the Franck-Condon point (labelled $\mathrm{S}_{0} \mathrm{Min}$ ) to the ts- $\mathrm{S}_{1}$ global minima. The conical intersection coordinates (CIX) are taken from Ref[17].

acetylene/benzene bond length and angles are shown in Fig 1 as a schematic of the energy change along this path. The potential surfaces along a vector joining the $1 \mathrm{~B}_{1 u}$ minima to ts- $\mathrm{S}_{1}$ minima using the $\mathrm{CAS}(10,10)$ level of theory is shown in Fig 2. The $2 \mathrm{~A}_{u}$ state exhibits a dramatic drop from several $\mathrm{eV}$ above the $\mathrm{B}_{2 u}$ at the $\mathrm{FC}$ point to become the global $\mathrm{S}_{1}$ minima.

CAS RS2 calculations are too expensive (especially without using symmetry) to obtain the large number of single point energies required to construct a vibronic coupling model, Since CASSCF energies give poor results compared to RS2 calculations and match poorly to experimental energies, the more efficient, DFT-MRCI method was used. This is a DFT based method for 

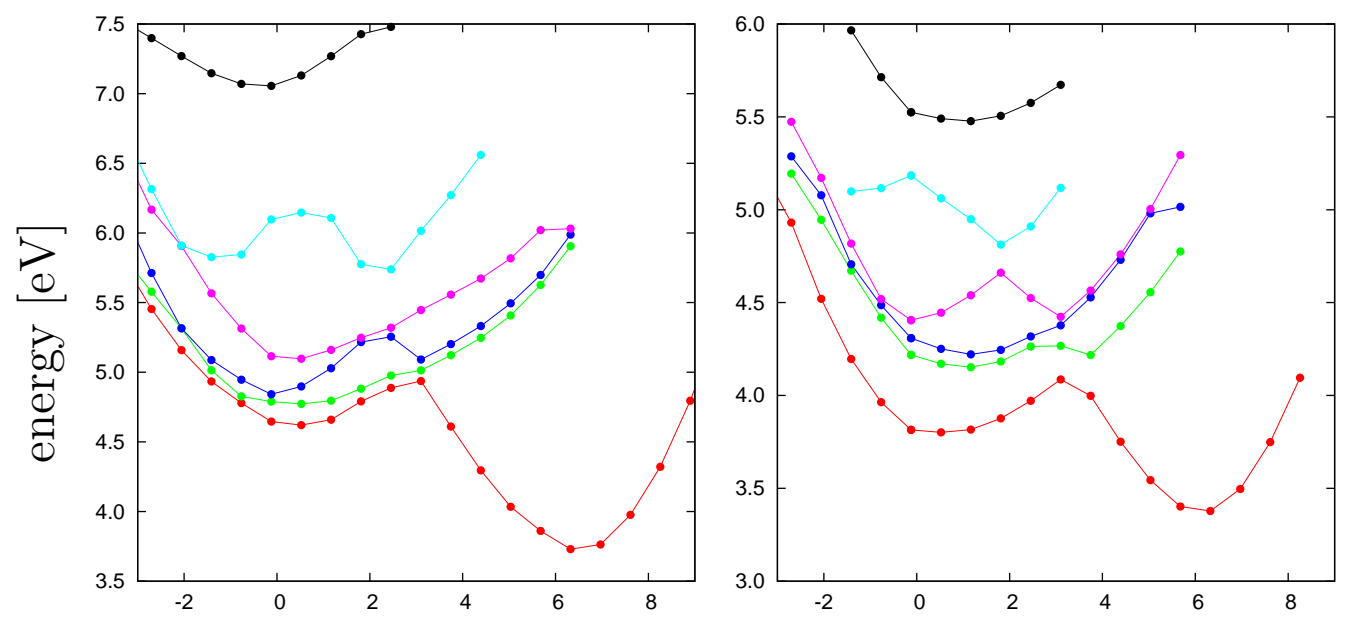

Figure 2: The PES along a vector connecting the $1 \mathrm{~B}_{1 u}$ minimum geometry to the ts- $\mathrm{S}_{1}$ geometry (left) CAS $(10,10)+\mathrm{RS} 2 \mathrm{C}$ (active space (g) in Table 2) and (right) DFT-MRCI ((f) in Table 2). Lines merely interpolate between points.

computing excited singlet and triplet states in conjugated molecules which includes both dynamic and static correlation (further details in [29]). Given that these most recent calculations place the Energies and states obtained from this method are given in column (f) of Table 2 .

The right cell in Fig 2 shows the cut through the PES from the $1 \mathrm{~B}_{1 u}$ minimum geometry to the ts- $\mathrm{S}_{1}$ minima obtained with DFT-MRCI. There is qualitative agreement between this method with the $\operatorname{CAS}(10,10)$ result. This method places $1 \mathrm{~B}_{1 u}$ as the $\mathrm{S}_{1}$ minima at the $\mathrm{FC}$ geometry, as tentatively suggested by experiments [9], but disagreeing with all multiconfigurational methods presented here (including dynamic correlation from RS2). It is nevertheless in agreement with $\mathrm{ADC}(2)$ cc-pVDZ calculations [18], TD-DFT 6-31G* calculations $[13,30,16]$ as well as calculations using semi-empirical hamiltonian $[7,15]$. This suggests that $1 \mathrm{~B}_{1 u}$ is the $\mathrm{S}_{1}$ state, and implies that the CAS+RS2 treatment of dynamic electron correlation does not result in a balanced description of the states. Some risk is taken by using a method that perhaps misplaces the first three excited states (albeit by a slight value); the mechanism for overcoming the isomerisation barrier might involve population transfer to state $\mathrm{B}_{3 g}$ or $\mathrm{B}_{2 u}$ (the latter considered a possibility [10]). However, experimental data [10] suggest the population between these do not freely equilibrate in condensed media and show little signs of being part of the mechanism leading to the trans-stilbene minima at room temperatures.

\subsection{Potential energy landscape and fitting.}

The normal mode coordinates used in the vibronic coupling model were obtained from a frequency calculation at the MP2/cc-pVDZ level using Gaus- 
sian 03 [31], at the ground-state optimized geometry at the same level of theory. There are 66 internal normal coordinates. Consequently, we focused our efforts in exploring the coordinates that will allow us to construct a model that is able to correctly describe population transfer $(\mathrm{PT})$ from $1 \mathrm{~B}_{1 u}$ to $2 \mathrm{~A}_{u}$. That is, we include totally symmetric coordinates (which have gradients of the potential energy at the FC point), coordinates that enable the description of the ts- $\mathrm{S}_{1}$ minima which, as we shall show, are $\mathrm{B}_{3 g}$ coordinates, and coordinates that can couple states $2 \mathrm{~A}_{u}$ and $1 \mathrm{~B}_{1 u}$, which have symmetry $\mathrm{B}_{1 g}$. In addition, we included odes of symmetry $\mathrm{B}_{2 g}$, which allowed us to represent a possibly important second order coupling mechanism, and of which we will have much to discuss below. The most relevant modes to this work are shown in Table 3.

The fact that we have only included modes that effectively couple the $2 \mathrm{~A}_{u}$ and $1 \mathrm{~B}_{1 u}$ states immediately implies it can only determine if IC via these states is relevant to the isomerisation mechanism, and obviously cannot address whether other channels are important to it. We shall have more to say on this in the discussion section 4 .

The system is known to undergo intersystem-crossing to triplet states with a life-time of hundreds of picoseconds and therefore does not undergo fast IC back to $\mathrm{S}_{0}$, and we found no $\mathrm{S}_{0} / \mathrm{S}_{1}$ intersections on the singlet state landscape examined. The ground state surface was only subsequently used to construct the initial nuclear wavepacket and is omitted from all figures below, to highlight the excited states more clearly. It is worth noting that the ground state potential was found to be adequately described by harmonic, or bounded Morse potentials for all modes. The MCTDH operator file for the model containing all the parameters is provided in supplementary information.

\subsection{1. trans-stilbene minima}

The model must first describe the potential surfaces connecting the FranckCondon point to the ts- $\mathrm{S}_{1}$ minimum. The ts- $\mathrm{S}_{1}$ minimum is principally described by displacement along coordinates of $\mathrm{B}_{3 g}$ and $\mathrm{A}_{g}$ symmetries; the vectors with largest displacements are shown in Table 4 . Totally symmetric modes of course cannot break the molecular symmetry and it is the $\mathrm{B}_{3 g}$ modes that are key in going to the trans structure and it can be seen that two $\mathrm{B}_{3 g}$ modes, $\tilde{v}_{5}$ and $\tilde{v}_{14}$, are particularly important. As will be seen below, distortions along these modes provide most of the stabilisation arising in the $2 \mathrm{~A}_{u}$ state. 


\begin{tabular}{|c|c|c|c|c|c|c|c|}
\hline$\#$ & $\mathrm{D}_{2 h}$ & e-vector & $\omega\left(\mathrm{cm}^{-1}\right)$ & $\#$ & $\mathrm{D}_{2 h}$ & e-vector & $\omega\left(\mathrm{cm}^{-1}\right)$ \\
\hline$\tilde{v}_{2}$ & $\mathrm{~B}_{2 u}$ & & 17.8 & $\tilde{v}_{20}$ & $\mathrm{~A}_{g}$ & & 703.2 \\
\hline$\tilde{v}_{4}$ & $\mathrm{~B}_{2 g}$ & & 122.6 & $\tilde{v}_{24}$ & $\mathrm{~B}_{1 g}$ & & 846.1 \\
\hline$\tilde{v}_{5}$ & $\mathrm{~B}_{3 g}$ & & 135.5 & $\tilde{v}_{29}$ & $\mathrm{~B}_{1 g}$ & & 950.2 \\
\hline$\tilde{v}_{6}$ & $\mathrm{~A}_{g}$ & & 257.2 & $\tilde{v}_{33}$ & $\mathrm{~A}_{g}$ & & 1008.4 \\
\hline$\tilde{v}_{7}$ & $\mathrm{~B}_{2 g}$ & & 258.9 & & $\mathrm{~A}_{g}$ & & 1160.0 \\
\hline & $\mathrm{B}_{1 g}$ & & 397.9 & & $\mathrm{~A}_{g}$ & & 1646.6 \\
\hline & $\mathrm{B}_{3 g}$ & & 514.5 & $\tilde{v}_{56}$ & $\mathrm{~A}_{g}$ & & 2216.1 \\
\hline$\tilde{v}_{17}$ & $\mathrm{~B}_{3 g}$ & & 623.4 & $\tilde{v}_{66}$ & $\mathrm{~A}_{g}$ & & 3246.0 \\
\hline
\end{tabular}

Table 3: $\mathrm{D}_{2 \mathrm{~h}}$ Harmonic frequencies of tolane (MP2/cc-pVDZ basis), important in this work.

\begin{tabular}{l|cccccc} 
& $\tilde{v}_{5}\left(\mathrm{~B}_{3 g}\right)$ & $\tilde{v}_{6}\left(\mathrm{~A}_{g}\right)$ & $\tilde{v}_{14}\left(\mathrm{~B}_{3 g}\right)$ & $\tilde{v}_{17}\left(\mathrm{~B}_{3 g}\right)$ & $\tilde{v}_{20}\left(\mathrm{~A}_{g}\right)$ & $\tilde{v}_{33}\left(\mathrm{~A}_{g}\right)$ \\
\hline CAS & 6.325 & 5.615 & -3.775 & -0.820 & 2.280 & 1.350 \\
Model & 4.829 & 6.261 & -5.735 & $\mathrm{~N} / \mathrm{A}$ & 2.066 & 0.251 \\
\hline & $\tilde{v}_{38}\left(\mathrm{~A}_{g}\right)$ & $\tilde{v}_{50}\left(\mathrm{~A}_{g}\right)$ & $\tilde{v}_{54}\left(\mathrm{~A}_{g}\right)$ & $\tilde{v}_{56}\left(\mathrm{~A}_{g}\right)$ & $\tilde{v}_{66}\left(\mathrm{~A}_{g}\right)$ & \\
\hline CAS & 1.176 & 0.698 & 0.569 & -3.343 & 0.662 & \\
Model & 0.812 & N/A & 0.138 & -2.780 & $\mathrm{~N} / \mathrm{A}$ &
\end{tabular}

Table 4: Ground state normal mode vectors leading from the FC geometry to the CASSCF optimized ts- $\mathrm{S}_{1}$ ("CAS") and model ts-S $\mathrm{S}_{1}$ optimized ("Model") geometries
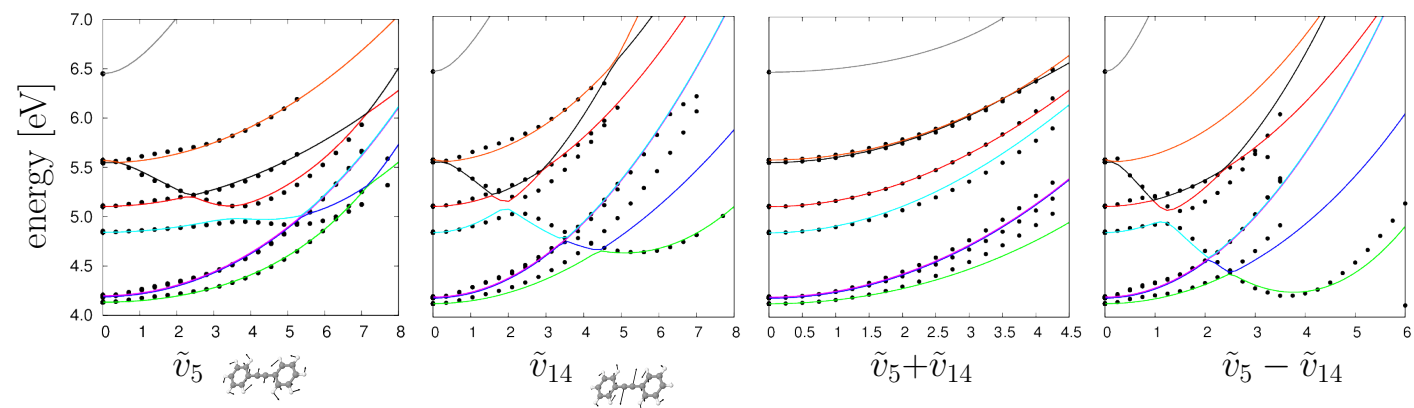

Figure 3: Cuts through the potential energy surfaces of tolane along modes of $\mathrm{B}_{3 g}$ symmetry. Solid lines adiabatic states from the fitted vibronic coupling model. Points are calculated at the DFT-MRCI level of theory. 
Fig 3 shows cuts along $\tilde{v}_{5}$ and $\tilde{v}_{14}$, as well as their diagonal and antidiagonal cuts (referring to quadrants in this 2D space). Eight states were calculated using DFT-MRCI; although state $2 \mathrm{~A}_{u}$ is $\mathrm{S}_{9}$ at the FC geometry, its energy is very close to $S_{7}$ and since we are only interested in the dynamics of low lying states, we only need to describe properly the $2 \mathrm{~A}_{u}$ stabilisation onto the ts- $\mathrm{S}_{1}$ geometry. Since it is along these two modes alone that stabilisation of $2 \mathrm{~A}_{u}$ occurs, we approximate the $2 \mathrm{~A}_{u}$ state along other coordinates as harmonic, with a force constant similar to the ground state, unless other monomials were required.

Notice the contrast between the diagonal and anti-diagonal cuts; there is a sharp gradient along the anti-diagonal direction. Our attempt to fit intrastate quadratic, quartic and bilinear polynomials to this $2 \mathrm{D}$ surface yielded a poor fit, Instead, a much more natural fit was achieved by assuming that stabilisation was caused by linear coupling to some higher lying state. The functional form of the adiabatic energies between these coupled states can be written as:

$$
W_{11}=\frac{D_{11}+D_{22}}{2}-\frac{\sqrt{\left(D_{22}-D_{11}\right)^{2}-4\left(\lambda_{5} \cdot Q_{5}+\lambda_{14} \cdot Q_{14}\right)^{2}}}{2}
$$

$\mathrm{D}_{11}$ and $\mathrm{D}_{22}$ refer to the intra-state elements of $2 \mathrm{~A}_{u}$ and the higher lying state, respectively and $\lambda_{5}$ and $\lambda_{14}$ to the inter-state coupling parameters along $\tilde{v}_{5}$ and $\tilde{v}_{14}$. This functional form gives adequately shaped surfaces near the $\mathrm{FC}$ region due to the linear terms entering the expansion. The state to which $2 \mathrm{~A}_{u}$ couples needs to be of $\mathrm{B}_{3 u}$ symmetry and DFT-MRCI situates the next state of that symmetry at $6.45 \mathrm{eV}$. This suggests the adiabatic state with the ts- $\mathrm{S}_{1}$ minimum is formed by the mixing of diabatic states with $\mathrm{A}_{u}$ and $\mathrm{B}_{3 u}$ character. This state was therefore included in the model, but with no adiabatic points to constraint the fit of the higher-lying adiabatic state along the modelled degrees of freedom; a zeroth order (harmonic oscillator) model was used, unless otherwise required.

Fig 4 shows cuts along the $\mathrm{A}_{g}$ modes. Of these $\tilde{v}_{6}, \tilde{v}_{38}$ and $\tilde{v}_{56}$ play a major role in the vector towards ts- $\mathrm{S}_{1}$. Terms correlating these coordinates were also included in the model, but are not shown here for clarity. Modes $\tilde{v}_{56}$ and $\tilde{v}_{54}$ show interesting crossings between many high lying states and might play an important role in dynamics at higher energies. Modes of $\mathrm{A}_{g}$ and $\mathrm{B}_{3 g} \otimes \mathrm{B}_{3 g}$ symmetry also enable first and second order coupling (respectively) between the $A_{u}$ states $\left(S_{4}\right.$ and $\left.S_{9}\right)$ and significant coupling can be inferred in 

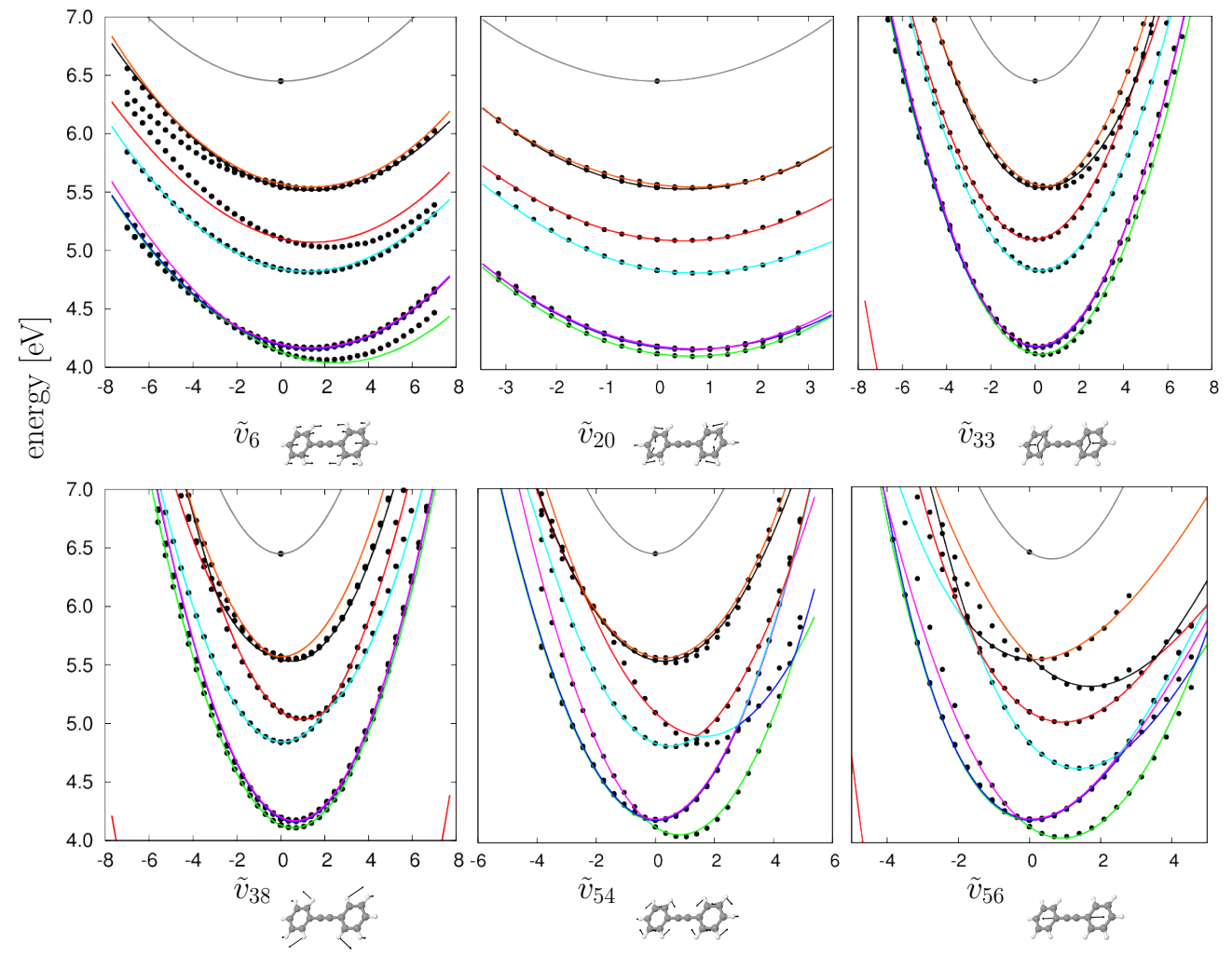

Figure 4: Fitting of DFT-MRCI potential surfaces of tolane along modes of $\mathrm{A}_{g}$ symmetry.

Fig 3. $1 \mathrm{~A}_{u}$ can then potentially play an important role as an intermediate state during the transfer of population from $1 \mathrm{~B}_{1 u}$ to $2 \mathrm{~A}_{u}$ via second order coupling modes of $\mathrm{B}_{3 g}$ symmetry (see section 3.3).

The ts- $\mathrm{S}_{1}$ minima is a highly correlated point in the normal coordinate basis, with small contributions from tens of modes. However, this region of space can be adequately described by the space of $3 \mathrm{~A}_{g} \oplus \mathrm{B}_{3 g}$ coordinates $\tilde{v}_{6}, \tilde{v}_{38}, \tilde{v}_{56}, \tilde{v}_{5}$ and $\tilde{v}_{14}$, and $2 \mathrm{D}$ polynomials were fitted along this region of space for combinations of these modes (Fig 5). To simultaneously fit these $2 \mathrm{D}$ subspaces, as well as the ts- $\mathrm{S}_{1}$ minima region, with a limited number of polynomials was only partially achieved, with the cost of a higher RMSD than on other spaces (attempts at fitting 3D polynomials complicated the fitting procedure). The fit to the vector joining the $\mathrm{B}_{1 u}$ minima to the ts$\mathrm{S}_{1}$ minima mentioned above is shown in Fig 6 , together with the fit along a vector displacing equally along each of the 5 coordinates involved in the 

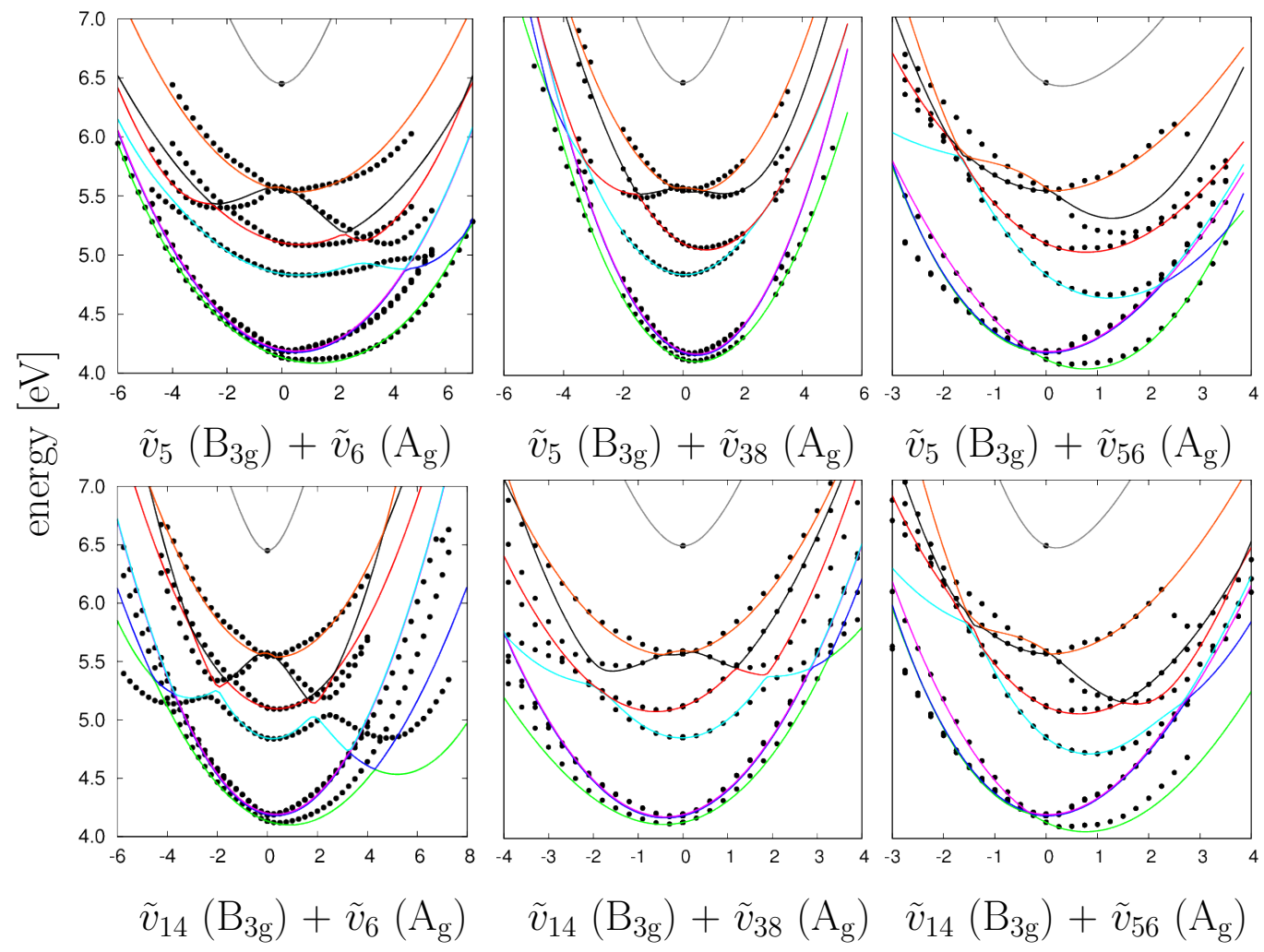

Figure 5: Fitting of DFT-MRCI potential surfaces of tolane along important combination modes of $\mathrm{A}_{g}$ and $\mathrm{B}_{3 g}$ symmetry.

model. The topography of the surfaces is seen to be modelled reasonably well, although the model curves rise too steeply and the lowest crossing points are displaced in energy and coordinate space.

By performing a local optimisation starting from a trans bent geometry, we were able to obtain an approximate model energy for the adiabatic $\mathrm{S}_{1}$ minimum of $3.04 \mathrm{eV}$, underestimating the experimental energies. We obtain $1.425 \AA$ for the acetylene benzene fragment bond length and $1.401 \AA$ for the acetylene bridge bond length, overestimating the latter compared to the electronic structure optimisation provided in Fig 1. The minimum in terms of normal modes in this $\mathrm{A}_{g} \oplus B_{3 g}$ subspace are given in Table 4. Note that the vectors in the table should not match exactly as we are fitting to DFT-MRCI energies and the ts- $\mathrm{S}_{1}$ minima vector was generated using a CASSCF geometry optimisation. The model obtains two identical minima 

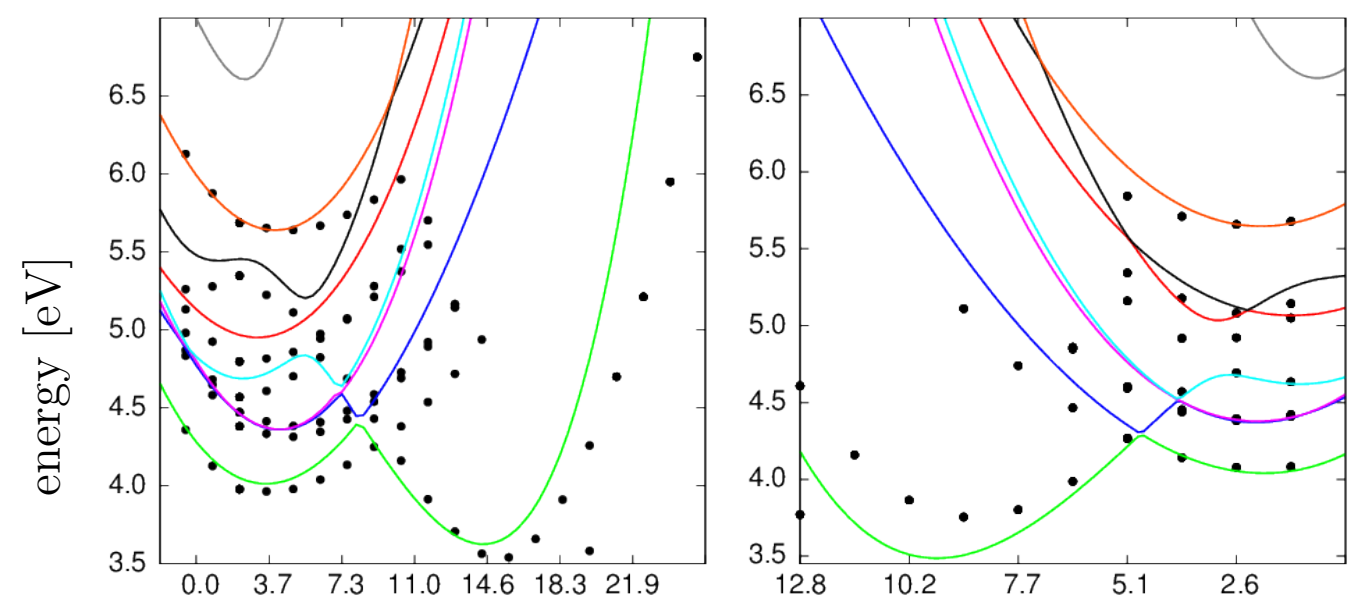

Figure 6: Cut through the tolane potential surfaces, with the Vibronic Coupling model fit as a solid line. (left) Along a vector joining the $\mathrm{B}_{1 u}$ minimum to the $\mathrm{ts}_{\mathrm{s}} \mathrm{S}_{1}$ structure. (right) Along a vector stepping equally along the five most important modes of $\mathrm{A}_{g}$ and $\mathrm{B}_{3 g}$ symmetry.

corresponding to either of the symmetrically equivalent trans geometries.

\subsection{2. $B_{1 u} / A_{u}$ state coupling.}

Having obtained surfaces which exhibit the qualitatively correct critical features for states $1 \mathrm{~B}_{1 u}$ and $2 \mathrm{~A}_{u}$, we are left with the task of understanding the coupling mechanisms that lead to the transfer of population. Modes $\mathrm{B}_{3 g}$ and $\mathrm{A}_{g}$, describing the isomerisation, do not allow for any linear coupling between states $1 \mathrm{~B}_{1 u}$ and $2 \mathrm{~A}_{u}$, not even indirectly via any of the intermediate states. Given the high symmetry of this molecule, we can explore a restricted number of coordinates which would allow, by symmetry considerations, any direct $\mathrm{B}_{3 g}$ and $\mathrm{A}_{g}$ coupling. Modes that allow linear coupling have $\mathrm{B}_{1 g}$ symmetry and are shown in the top of Fig 7 ; there is little evidence of any direct coupling between our states of interest along any single $\mathrm{B}_{1 g}$ cut. This is clearly a result of the large energy separation between these states at the FC region. Therefore, we get a better estimate of coupling by exploring cuts that include the $\mathrm{B}_{3 g}$ subspace, as these enable states to get close and exhibit any coupling effects near the crossing region. Fig 7 also depict cuts along the $\tilde{v}_{5}+\tilde{v}_{14}\left(\mathrm{~B}_{3 g}\right)$ diagonal with the three $\mathrm{B}_{1 g}$ modes. Third order terms were also fitted along these modes. The estimated linear/third order coupling, implicitly inferred via the gap of the $\mathrm{A}_{u} / \mathrm{B}_{1 u}$ avoided crossing, is clearly present (bottom of Fig 7). It is worth noting that the kinks observed near the crossing along the three dimensional vector cuts are a result of a higher lying state affecting the electronic structure calculation. These kinks might express themselves in a increased estimation of the linear coupling between 

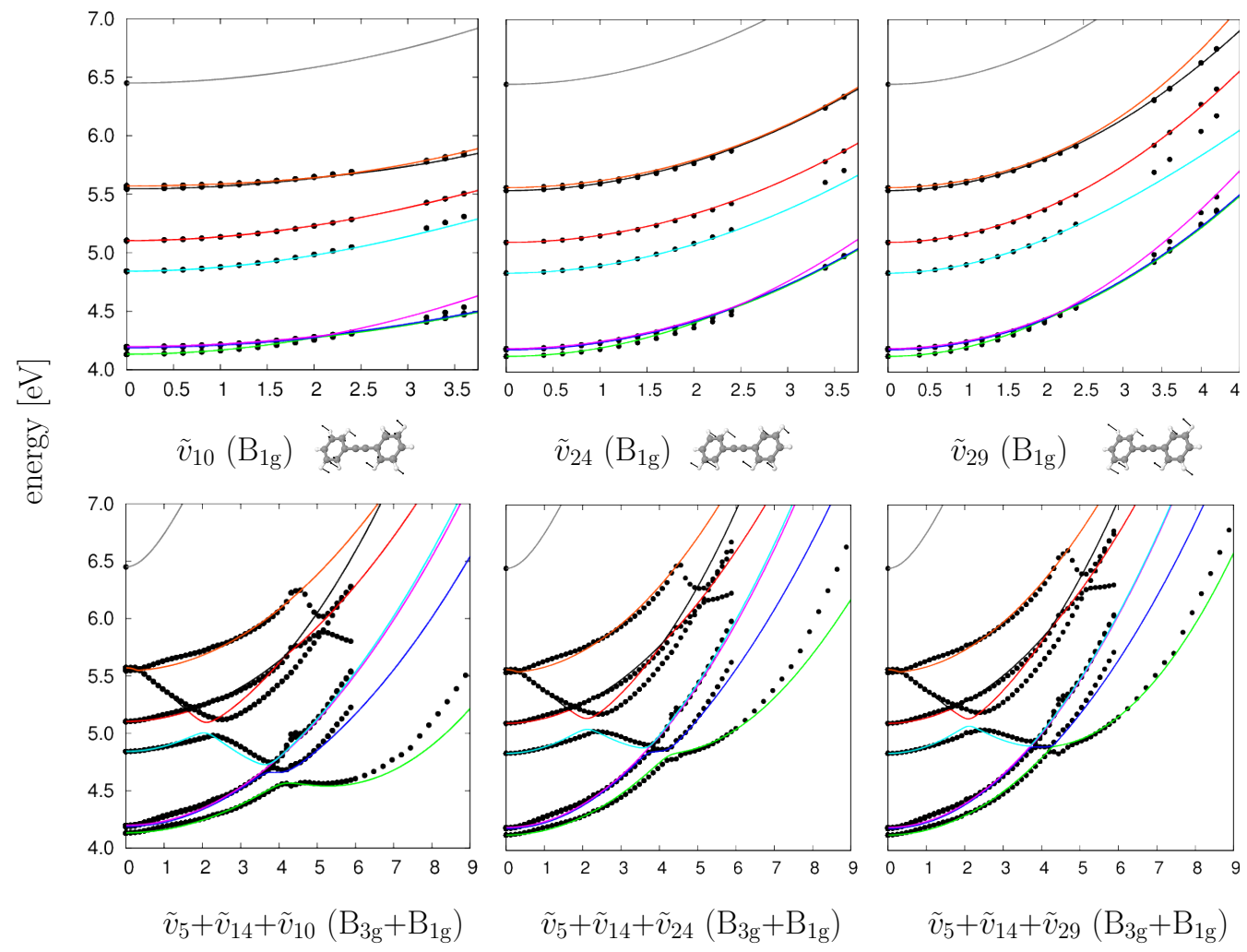

Figure 7: Fitting of surfaces along modes of $\mathrm{B}_{1 g}$ and $\mathrm{B}_{3 g}$ symmetry (enabling linear and third order coupling).

these states. But, as we will show below, even this potential overestimation is still insufficient to make these modes alone drive the IC mechanism.

In turn, we investigate if second-order coupling might be playing a role in the transfer of population. To obtain second-order coupling between $1 \mathrm{~B}_{1 u}$ and $2 \mathrm{~A}_{u}$ but simultaneously reduce the energy separation between these states, coordinates are needed that couple while simultaneously displacing along $\mathrm{B}_{3 g}$ modes $\left(\tilde{v}_{5}\right.$ or $\left.\tilde{v}_{14}\right)$. Thus we have to examine coordinates of symmetry $\mathrm{B}_{1 g} \otimes \mathrm{B}_{3 g}=\mathrm{B}_{2 g}$. Fig 8 shows $2 \mathrm{D}$ cuts along two coordinates, $\tilde{v}_{4}$ and $\tilde{v}_{7}$, of $\mathrm{B}_{2 g}$ symmetry which exhibit signs of coupling, by the presence of a shoulder on $1 \mathrm{~B}_{1 u}$, between states $1 \mid 2 \mathrm{~A}_{u}$ and $1 \mathrm{~B}_{1 u}$ when simultaneously displacing along $\tilde{v}_{5}$ or $\tilde{v}_{14}\left(\mathrm{~B}_{3 g}\right)$. Also notable is the mild double well present for state $1 \mathrm{~A}_{u}\left(\mathrm{~S}_{4}\right)$ along the $\mathrm{B}_{2 g}$ modes (left plots of Fig 8) which cross the $1 \mathrm{~B}_{1 u}\left(\mathrm{~S}_{1}\right)$ state. Together with the first and second coupling between $\mathrm{A}_{u}$ states $\left(\mathrm{S}_{4}\right.$ and 

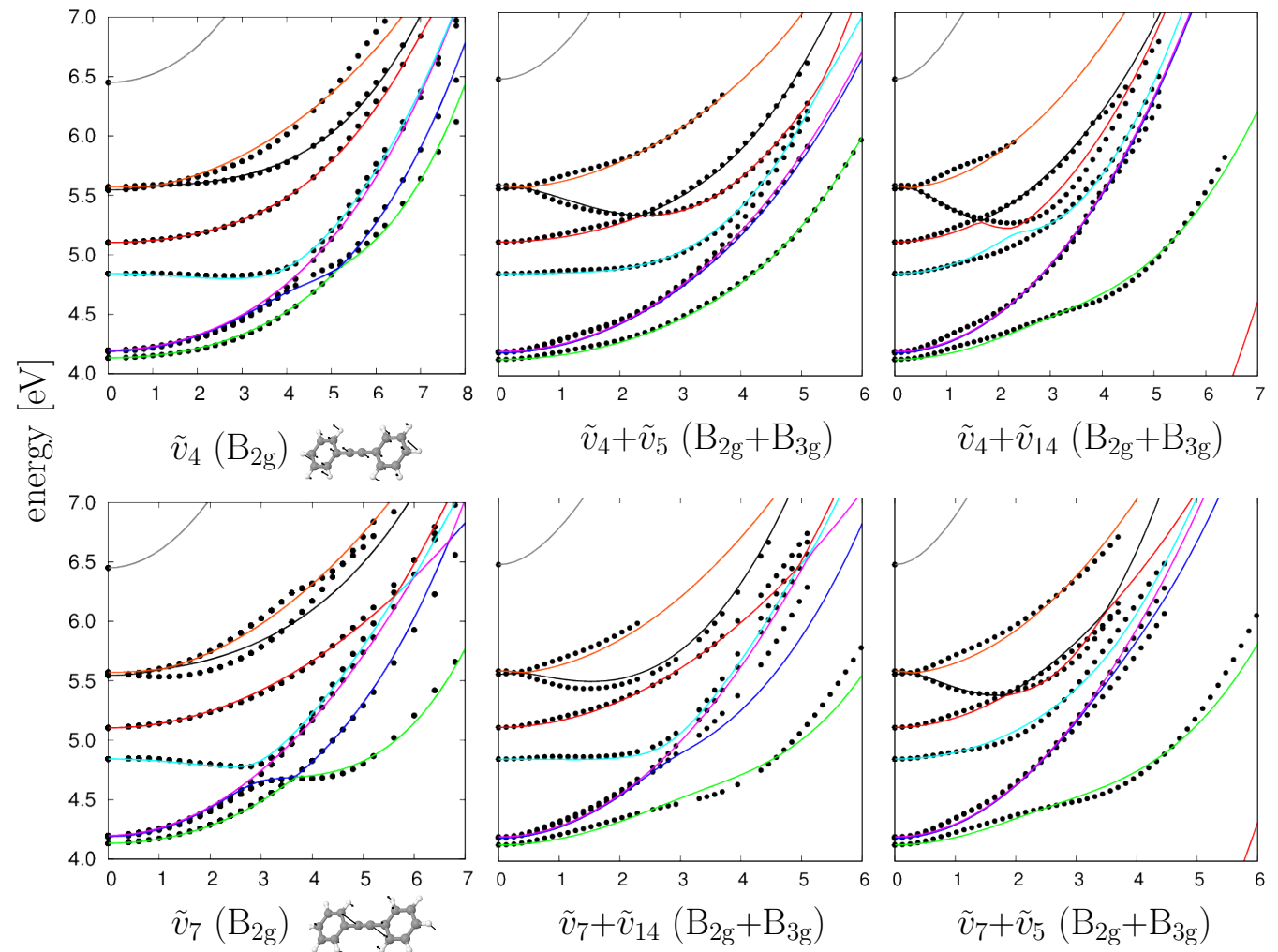

Figure 8: Fitting of surfaces along modes of $\mathrm{B}_{2 g}$ and $\mathrm{B}_{3 g}$ symmetry (enabling second order coupling).

$\mathrm{S}_{7}$ ) along modes of $\mathrm{A}_{g}$ and $\mathrm{B}_{3 g} \times \mathrm{B}_{3 g}$ symmetry (mentioned above), state $1 \mathrm{~A}_{u}\left(\mathrm{~S}_{4}\right)$ can transfer population between $2 \mathrm{~A}_{u}$ and $\mathrm{B}_{1 u}$. In section 3 we shall show that these modes, corresponding to an out-of-plane bending, play a more significant role during the energy transfer observed in the propagation.

\section{Dynamics}

Using the vibronic coupling model Hamiltonian set up above, quantum dynamics simulations can be run to simulate the IC and analyze the modes and states involved. First the model must be validated by calculating the absorption spectrum. 


\subsection{MCTDH numerical details}

To perform wavepacket propagation we used the MCTDH algorithm [27]. The normal mode coordinates were combined together to provide four particles as follows: modes of $\mathrm{B}_{3 g}$ symmetry $\left(\tilde{v}_{5 \mid 14}\right)$, the most correlated of $\mathrm{A}_{g}$ symmetry $\left(\tilde{v}_{6|38| 56}\right)$, the linear coupling modes of $\mathrm{B}_{1 g}$ symmetry $\left(\tilde{v}_{10|24| 29}\right)$ and the quadratic coupling modes of $\mathrm{B}_{2 g}$ symmetry $\left(\tilde{v}_{4 \mid 7}\right)$. We typically used at least 5 single particle functions $(\mathrm{SPF})$ for the $\mathrm{S}_{1}, \mathrm{~S}_{7}$ and $\mathrm{S}_{8}\left(\mathrm{~B}_{1 g}\right.$ and $\mathrm{A}_{u}$ states) and at least 3 for the other states. Hermite discrete variable representation (DVR) functions were chosen as spectral basis, with 25 DVR points on all modes but $\tilde{v}_{5}, \tilde{v}_{14}$, and $\tilde{v}_{56}$, which had 35 . The spectra were calculated by Fourier transforming the autocorrelation function. For IC to the ts- $\mathrm{S}_{1}$ geometry, only states $1 \mathrm{~B}_{1 u}, 1 \mathrm{~A}_{u}, 2 \mathrm{~A}_{u}$ and $1 \mathrm{~B}_{3 u}\left(\mathrm{~S}_{1}, \mathrm{~S}_{4}, \mathrm{~S}_{7}\right.$ and $\left.\mathrm{S}_{8}\right)$ were propagated, since these are principally involved in the population transfer along the modes included in the model.

\subsection{Absorption spectra}

There is some uncertainty as to the precise energy ordering of the first three excited states. To help answer this question the absorption spectrum was calculated using the model and matched to the experimental spectra at both high and low temperatures. Since the model contains only terms that only very weakly couples $1 \mathrm{~B}_{1 u}$ to $1 \mathrm{~B}_{2 u}$ and none that couple to $1 \mathrm{~B}_{3 g}$, we shifted the vertical excitation energies to be those suggested by either the SACAS $(12,12)+$ RS2C or the DFT-MRCI determined energies (Table 2). The $1 \mathrm{~B}_{1 u}$ state is the significant bright state in the energy range shown in the absorption spectra, with $1 \mathrm{~B}_{2 u}$ also having a small oscillator strength (section 2.1). To examine the importance of the darker states, we also varied the population projected from the ground state to the $1 \mathrm{~B}_{3 g} / 1 \mathrm{~B}_{2 u}$ states. Figure $\mathrm{S} 1$ provided as supplementary information show the spectra from these tests, using the modes in the 'full' model (label referring to table in Fig 10 ).

The top row of Fig 9 shows the experimental spectra at $77 \mathrm{~K}$ and 298 $\mathrm{K}$ (taken from ref. [32] and [6] respectively) and the bottom row of Fig 9 shows the calculated spectrum with two different excitation ratios and the states placed according to the energies from the DFT-MRCI calculations: the $\mathrm{CAS}(12,12)+\mathrm{RS} 2 \mathrm{C}$ energies provided poor modelled spectra.

The calculated spectrum that best resembles the room temperature exper-

imental spectrum has $30 \%$ of the population initially transferred to the $1 \mathrm{~B}_{2 u}$ and $1 \mathrm{~B}_{3 g}$ states, while the spectrum best resembling the low-temperature experimental spectrum has $0 \%$ population entering states $1 \mathrm{~B}_{2 u}$ and $1 \mathrm{~B}_{3 g}$ 
(the $15 \%$ is nearly as good). The calculated spectra have near-quantitative agreement between the principal peaks.

That both high and low temperature spectra with the DFT-MRCI energies for states $1 \mathrm{~B}_{2 u}$ and $1 \mathrm{~B}_{3 g}$ match the experimental spectra more closely suggests $1 \mathrm{~B}_{1 u}$ to be the first excited state. That little transfer of population to states $1 \mathrm{~B}_{2 u}$ and $1 \mathrm{~B}_{3 g}$ occur at low temperature can be expected, as modes that might couple such states would not be significantly excited. It is worth noting that the spectra obtained from the models with DFT-MRCI placed energies, only significantly differ in the relative height of the four most prominent peaks. The spectra are predominantly a result of the $1 \mathrm{~B}_{1 u}$ state around the FC geometry, as this can be deduced from the spectra with $0 \%$ population to the $1 \mathrm{~B}_{2 u} / 1 \mathrm{~B}_{3 g}$ state matching closely the experimental one. Furthermore, in the 500 fs of propagation only $\sim 1 \%$ of the density was transferred to the higher lying states. This is also consistent with vibronic spectra already published in the literature [30, 13].

By obtaining a spectrum with $100 \%$ on $1 \mathrm{~B}_{2 u} / 1 \mathrm{~B}_{3 g}$ we concluded that peaks around 286-289 and 278-281 nm have a significant contribution from these states, and might be responsible for the change in relative heights between the two principal peaks observed in the experimental spectra at low/hi temperatures (shown in Fig 9). This spectra with no population onto $1 \mathrm{~B}_{1 u}$ results in a very poor match with the experimental one, further solidifying the identification of this state as the bright state which largely determines the experimental spectra.

The absorption spectra for different sets of reduced dimensional models at 'high' temperatures (left cell of Fig 9) were also calculated in order to help us analyze the origin of the progressions (Fig 10). As expected, from this figure we can see that the final spectra are principally built from vibrational progressions arising from totally symmetric modes, labeled $\mathrm{a}_{g} 1$, with some small contribution from modes labeled $b_{3 g}$ (table in Fig 10).

\subsection{Population transfer to $2 A_{u}$}

Experiments suggests that the dual fluorescence exhibited by tolane corresponds to the isomerization from ground state configuration to the transstilbene like geometry due to the strong stabilisation of the $2 \mathrm{~A}_{u}$ state.

It is also known that this process is susceptible to temperature - shutting down at low temperatures and competing with vibrational cooling at room temperature. This implies that population transfer between these states must be on similar timescales as those of vibrational energy relaxation, which is 

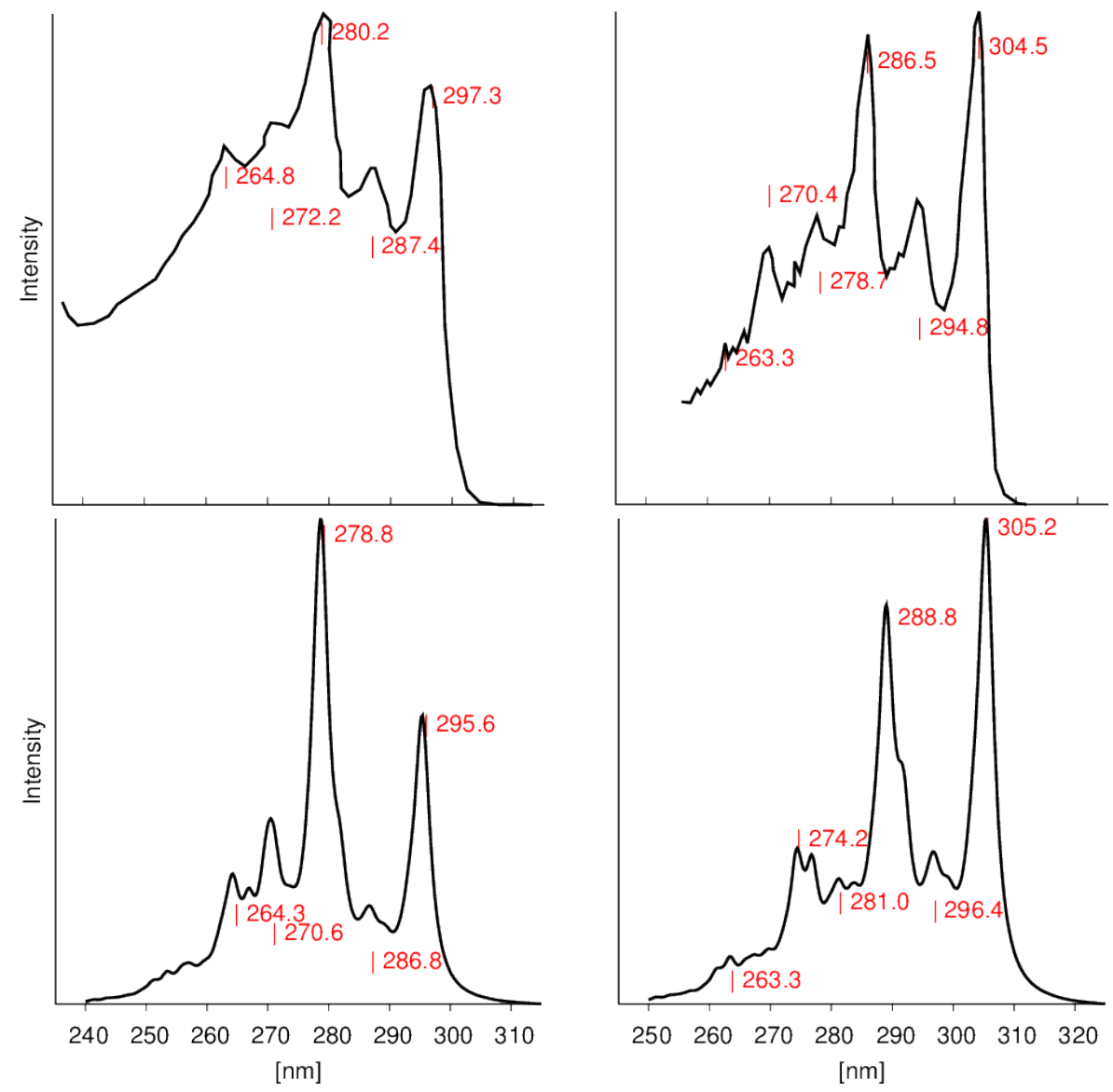

Figure 9: (bottom row) Calculated spectra with different populations in states $\mathrm{B}_{3 g}$ and $\mathrm{B}_{2 u}$ : (left) $30 \%$ and (right) 0\%. (top row) Experimental spectra (left) at high [6] and (right) low temperature [32]. The calculated spectra have been shifted to the red to match the experimental ones. Broadening has been used to approximately match the experimental spectra (a more resolved spectra is provided as supplementary information).

known to occur from hundreds of femtoseconds [33] to a few picosecond [34] timescales. Since no transfer occurs at low temperatures, this transfer must be due to wavepacket components arising from higher vibrational states being populated in the ground electronic state prior to absorption. The minimal model described above should therefore be able to describe the two experimental observations just mentioned. If we project the fully relaxed ground state wavefunction corresponding to a $0 \mathrm{~K}$ state (to compare with $77 \mathrm{~K}$ in the experiment [32] in Fig 9) we should not observe any transfer occurring. On the other hand, preparing a wavefunction with vibrational modes excited at room temperature should lead to transfer of energy on the femtosecond to picosecond timescales. 


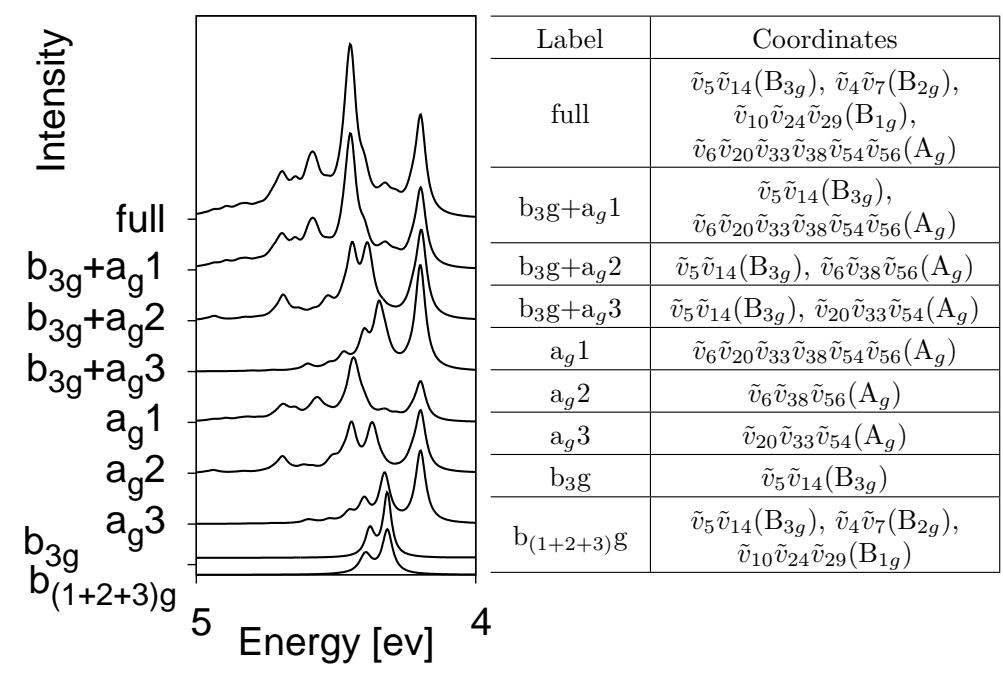

Figure 10: Comparing full spectra with different sets of reduced dimensionality spectra (left). Labels and coordinates of the different reduced dimensional models used to calculate absorption spectra are provided in the table.

Population transfer away from $1 \mathrm{~B}_{1 u}$ can only be expected to occur between states with comparable energies, namely $1 \mathrm{~B}_{2 u}, 1 \mathrm{~B}_{3 g}$ and ts- $\mathrm{S}_{1}$. However, the minimal model constructed does not have the coordinates, and therefore coupling coefficients, to adequately determine the transfer to states $1 \mathrm{~B}_{2 u}$ and $1 \mathrm{~B}_{3 g}$, but we shall be able to qualitatively determine the extent and mechanism of the direct coupling to ts- $\mathrm{S}_{1}$. Consequently, only four states, $1 \mathrm{~B}_{1 u}, 1 \mathrm{~A}_{u}, 2 \mathrm{~A}_{u}$ and $1 \mathrm{~B}_{3 u}\left(\mathrm{~S}_{1}, \mathrm{~S}_{4}, \mathrm{~S}_{7}\right.$ and $\left.\mathrm{S}_{8}\right)$ are involved in the modeling of this process. Since we do not include any dissipative effects in our model, we do not expect the entire population to transfer to the trans-stilbene $2 \mathrm{~A}_{u}$ state, but an eventual equilibration between the populations.

To determine the principal mechanism by which the transfer of population occurs, we can selectively switch the different coupling modes or states. To probe the issues just discussed we ran the following calculations:

- Using a fully ground-state relaxed initial wavefunction (0K wavepacket).

- Using a hot wavefunction, with some ground state, low frequency modes vibrationally excited, mimicking a room temperature wavepacket.

- Using a hot wavefunction as above, without $\mathrm{B}_{1 u} / \mathrm{A}_{u}$ linear coupling modes represented (symmetry $\mathrm{B}_{1 g}$ modes $\tilde{v}_{10|24| 29}$ ).

- Using a hot wavefunction as above, without $\mathrm{B}_{1 u} / \mathrm{A}_{u}$ second order coupling modes represented (symmetry $\mathrm{B}_{2 g}$ modes $\tilde{v}_{4 \mid 7}$ ).

- Using a hot wavefunction as above, without state $1 \mathrm{~A}_{u}$ represented, $\left(\mathrm{S}_{4}\right)$ to determine its importance as an transient state. 
The 'hot' wavepacket is built with modes $\tilde{v}_{4}\left(\mathrm{~B}_{2 g}\right), \tilde{v}_{5}\left(\mathrm{~B}_{3 g}\right)$ and $\tilde{v}_{6}\left(\mathrm{~A}_{g}\right)$ in their second vibrational excited state and modes $\tilde{v}_{7}\left(\mathrm{~B}_{2 g}\right), \tilde{v}_{10}\left(\mathrm{~B}_{1 g}\right)$ and $\tilde{v}_{14}\left(\mathrm{~B}_{3 g}\right)$ in their first vibrational excited state. Therefore, the only linear coupling mode excited is $\tilde{v}_{10}$, as opposed to the second order coupling modes $\tilde{v}_{4}$ and $\tilde{v}_{7}$. It is worth noting that the relative populations of the $\tilde{v}_{4}$ and $\tilde{v}_{10}$ vibrational states at room temperature is $\exp \left[-\beta\left(2 \omega_{10}-3 \omega_{4}\right)\right] \approx 0.12$, nearly an order of magnitude less populated.

Figure 11 shows the state-populations of the five propagations up to four picoseconds. It's worth noting that states $2 \mathrm{~A}_{u}$ and $1 \mathrm{~B}_{3 u}$ (shown blue and brown in plots) diabatically represent the adiabatic state that becomes ts- $\mathrm{S}_{1}$. As expected, the 'cold' wavepacket exhibits minimal transfer, mostly to $1 \mathrm{~A}_{u}$ $\left(\mathrm{S}_{4}\right)$. In contrast the 'hot' wavefunction exhibits a rapid 6-8\% transfer away from $1 \mathrm{~B}_{1 u}$ in the first hundred femtoseconds, followed by a slower picosecond timescale transfer to the ts-S1 states $\left(2 \mathrm{~A}_{u}\right.$ and $\left.1 \mathrm{~B}_{3 u}\right)$ and little to $1 \mathrm{~A}_{u}$.

We attribute the rapid transfer to the higher vibrational eigenfunction components populated when we project the ground state wavefunction under the sudden approximation. The right cells of this figure correspond to the last three items in the above list, excluding modes/states in order to understand their effect on the propagation. Excluding linear coupling parameters (labeled "no lin") has little effect on the ps timescale transfer away from $1 \mathrm{~B}_{1 u}$, but it slows down the transfer to $2 \mathrm{~A}_{u}$ on the first picosecond, and limits population to $1 \mathrm{~B}_{3 u}$ throughout. More population is initially transferred to $1 \mathrm{~A}_{u}$, but mostly stops after the first picosecond.

Not including second order coupling (labeled "no quad") reduces significantly transfer to states $2 \mathrm{~A}_{u} / 1 \mathrm{~B}_{3 u}$ (ts- $\mathrm{S}_{1}$ ), with most population transferred to $1 \mathrm{~A}_{u}$. Since reduced transfer occurs to $1 \mathrm{~B}_{3 u}$ when either first or second order coupling is absent, these two modes must together be working to enable the picosecond $1 \mathrm{~B}_{1 u}$ to $1 \mathrm{~B}_{3 u}$ transfer observed.

Finally, $1 \mathrm{~A}_{u}\left(\mathrm{~S}_{4}\right)$ has a slower but continuous transfer to states $2 \mathrm{~A}_{u} / 1 \mathrm{~B}_{3 u}$ which suggests that, although it is playing a role in IC to ts- $\mathrm{S}_{1}$, it is not indispensable to the process. Its absence also reduces the population transferred to $1 \mathrm{~B}_{3 u}$ (plot "no $1 \mathrm{~A}_{u}$ ").

\section{Discussion and conclusions}

A reduced-dimensionality vibronic coupling model Hamiltonian using DFTMRCI calculations has been set up to describe the photo-excited dynamics of tolane. 


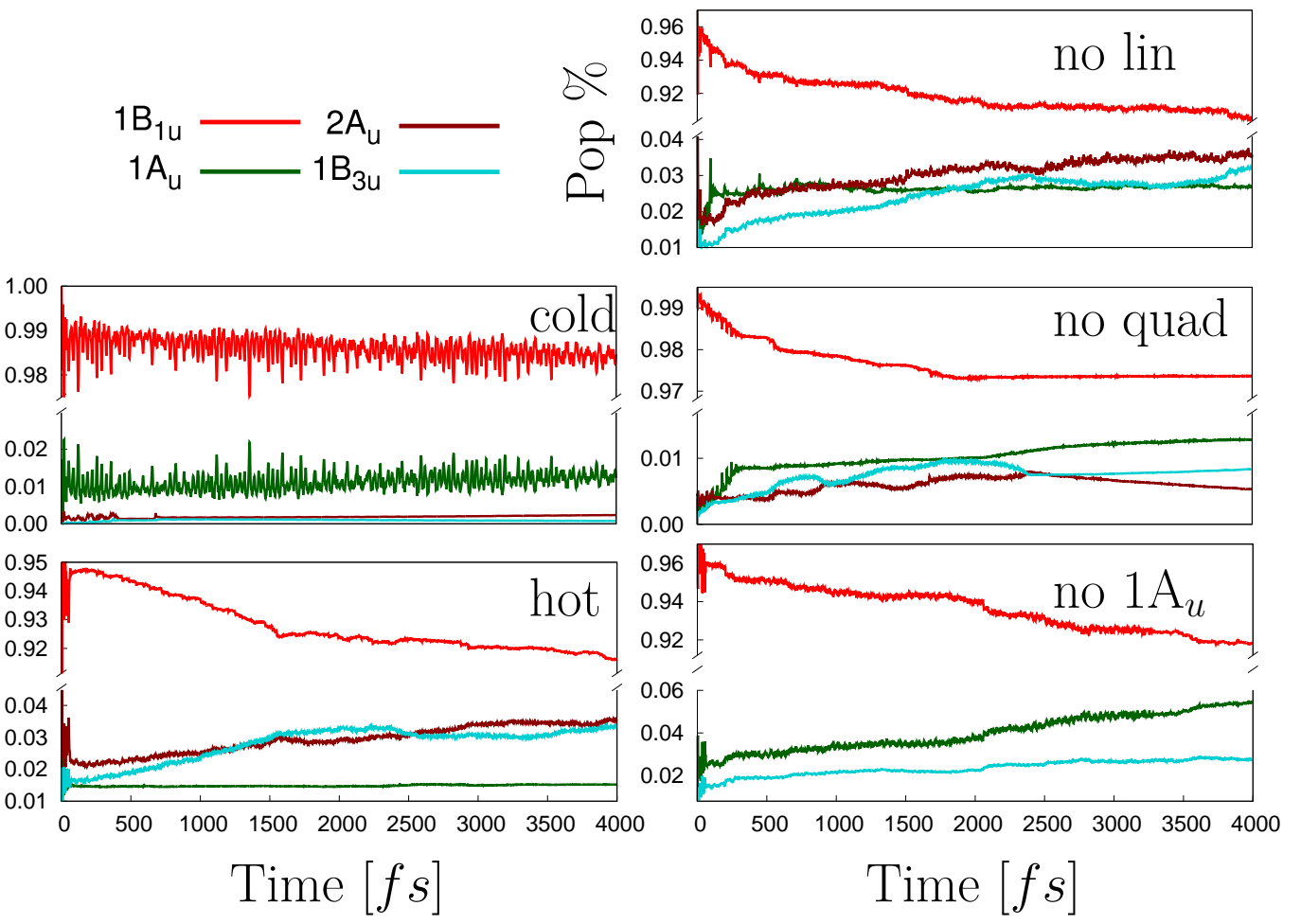

Figure 11: Population of diabatic states involved in the IC to the ts- $\mathrm{S}_{1}$ minima. Note that $2 \mathrm{~A}_{u}$ and $1 \mathrm{~B}_{3 u}$ together represent the adiabatic ts- $\mathrm{S}_{1}$ state. hot: ground state $\tilde{v}_{4-10}$ modes are initially excited (see text), cold: relaxed ground state as initial wavepacket, no lin: $1 \mathrm{~B}_{1 u} 2 \mathrm{~A}_{u}$ linear coupling terms excluded, no quad: $1 \mathrm{~B}_{1 u} 2 \mathrm{~A}_{u}$ second order terms excluded, no $1 A_{u}$ : state $1 \mathrm{~A}_{u}\left(\mathrm{~S}_{4}\right)$ excluded.

The first question to be addressed is the ordering of states. CASSCF $+\mathrm{RS} 2 \mathrm{C}$ methods suggest the energy ordering of the first three excited states at the Frank Condon geometry to be $\mathrm{B}_{3 g}, \mathrm{~B}_{2 u}, \mathrm{~B}_{1 u}$ (in order), with the DFT-MRCI placing $\mathrm{B}_{1 u}$ as $\mathrm{S}_{1}$. Given the agreement between the DFT-MRCI calculations with $\operatorname{ADC}(2)$ and TDDFT $[18,13]$, it is likely that $\mathrm{B}_{1 u}$ is the $\mathrm{S}_{1}$ state. This implies that CASSCF+RS2C, even with 12 orbitals in the active space, is unable to provide a balanced description for the excited states of these strongly correlated systems.

By calculating a set of different spectra under different conditions, a good match to the experimental spectra is found using the MRCI-DFT energies, and not the CASSCF+RS2C ones. For the room temperature spectra, the 
best fit was obtained where $\sim 30 \%$ of the excited state population is found in the dark $\mathrm{B}_{3 g}$ and $\mathrm{B}_{2 u}$ states, with these states lying higher than $\mathrm{B}_{1 u}$. This also supports the DFT-MRCI energy ordering of the states, as well as suggesting that some density is transferred to states. The oscillator strength of $1 \mathrm{~B}_{2 u}$ is small but present ( 0.05 au using $\operatorname{CAS}(10,10)$ ) and it can contribute to the spectra even at low temperatures.

The second question to be addressed is the pathways available for the isomerisation made by crossing from the linear $\mathrm{B}_{1 u}$ state to the trans ts- $\mathrm{S}_{1}$ state. Projecting the $\mathrm{B}_{1 u}-\mathrm{ts}_{\mathrm{s}} \mathrm{S}_{1}$ vector into the basis of normal coordinates tells us that the distortion predominantly involves $\mathrm{A}_{g}$ and $\mathrm{B}_{3 g}$ modes. Interestingly, the ts- $\mathrm{S}_{1}$ minimum is due to stabilisation of the high lying $2 \mathrm{~A}_{u}$ state, which was found to be more appropriately described by diabatically coupling to a higher lying state of $\mathrm{B}_{3 u}$ symmetry, rather than to a quartic and quadratic inter-state polynomial terms.

In agreement with Saltiel et al, we find that $\mathrm{IC}$ from the bright $1 \mathrm{~B}_{1 u}$ state to $1 \mathrm{~B}_{2 u}$ is likely to be slow and will therefore not compete with $\mathrm{IC}$ to the ts- $\mathrm{S}_{1}$ state. This can be seen by inspection of the PES cuts along the $\mathrm{B}_{3 g}$ modes in Fig 3 and the lack of linear coupling between these 2 states: where these two states cross shows no signs of linear coupling.

The potential surfaces from the calculated vibronic coupling model fit reasonably well the ab initio calculated surfaces for the configurations along the isomerisation path. The coupling between the involved states is present, but weak. Starting with a cold wavepacket no isomerisation IC is seen, while only a small amount of slow transfer is seen starting with a hot wavepacket in which the relevant low frequency modes were excited in the ground state. This is in line with experimental observations $[10,14]$. However, the rate of transfer is significantly slower than the 8 ps measured half-life [6], and this discrepancy can be due to a number of reasons.

Firstly, the description of the potential energy to the ts- $\mathrm{S}_{1}$ minima is only approximately achieved by the model and we can therefore only expect qualitative agreement with experiment. The electronic structure calculations may not be accurate, and even if they are the conical intersection in the model might be too high, maybe resulting in a lack of coupling. Alternatively, it might be that the mechanism to IC occurs via states $\mathrm{B}_{2 u}$ or $\mathrm{B}_{3 g}$, and the model might be missing modes (like $\mathrm{B}_{3 u}$ ) that couple these to $2 \mathrm{~A}_{u}$ (ts- $\mathrm{S}_{1}$ ), although, as mentioned above, we have not inferred any significant $\mathrm{B}_{2 u} / \mathrm{B}_{1 u}$ coupling and it has been suggested previously not to be significantly coupled $[15,10]$. Finally, to properly model the IC to ts- $\mathrm{S}_{1}$ at room temperature, 
one would have to average the results of all possible initial wave-packets determined by the density matrix, which would require huge computational resource and is outside the scope of the present study.

Assuming that the model is qualitatively correct, we can note that it suggests that second order coupling, and not first order coupling, to be the major driving force in the modes coupling $1 \mathrm{~B}_{1 u} / 2 \mathrm{~A}_{u}$. Nevertheless, we found a reduction of population transferred to $1 \mathrm{~B}_{3 u}$ (one of the two states making up ts- $\mathrm{S}_{1}$ ) if either is missing.

The effect of fitted linear coupling terms for $\tilde{v}_{10}$ and $\tilde{v}_{24}$ can be discerned in the lower cells in Fig 7, and appear adequate. The values are -0.011 and 0.049 respectively, small but not insignificant, and a case could be made that this would explain the long time-scales observed. It could therefore be that first order coupling still plays a significant role, with mode $\tilde{v}_{10}$ thermally excited to higher vibrational states in the ground state, and leading to transfer of population via the $\mathrm{A}_{g}$ and $\mathrm{B}_{1 g}$ tuning modes (likely $\tilde{v}_{6}$ and $\left.\tilde{v}_{5}\right)$. Nevertheless, if one concedes that higher vibrational excitations of these modes to be involved, it would also imply a stronger contribution from the second order coupling modes, having a lower force constant. Furthermore, second order coupling is also more naturally expected to exhibit a picosecond timescale transfer than a first order coupling term. This is apparent from the "no quad" cell in Fig 11, where only linear (and small third order) coupling terms are driving the IC, showing that all population transfer occurs during the first two picoseconds.

A further consideration is that these linear coupling modes $\left(\mathrm{B}_{1 g}\right)$ correspond to out-of-plane 'wobbles' of the benzene ring's hydrogen atoms (see Table 3), and would a-priori seem to have little involvement on the transstilbene distortion taking place along the acetylene bridge. In contrast, the trans, out-of-plane vibrations of the second order coupling modes $\left(\mathrm{B}_{2 g}\right)$ occur principally along this bridge. It would not be surprising that these are playing a role during the transfer process, since the orbitals characterising $1 \mathrm{~B}_{1 u}$ and $2 \mathrm{~A}_{u}$ are out-of-plane and in-plane acetylene $\pi \pi^{*}$ excitations.

Although it has been suggested that the conical intersection and main pathway to the ts- $\mathrm{S}_{1}$ occurs via the planar geometry $[18,17]$, that the first and second-order modes coupling states $1 \mathrm{~B}_{1 u}$ and $2 \mathrm{~A}_{u}$ contain out-of-plane distortions would suggest that the derivative coupling vector at the CIX would contain out-of-plane contributions, a question we were unable to directly address.

Tolane is the monomer of a family of phenyl-acetylene dendrimers which, 
owing to their efficient energy funnelling properties, could potentially be used to develop many applications involving light charge interconversion. It is therefore of some importance to have a thorough understanding of the mechanisms by which this monomer transfers energy. Kleiman et al[14] observed that the longer lived fluorescence (150-200 ps) arising from the ts- $\mathrm{S}_{1}$ state is missing in one of the dendrimers referred to as a nano-star, but the short lived fluorescence band is still present. It has been shown these dendritic antenna have states that are localized along the meta-deconjugated phenyl-acetylene branches. One suggested mechanism could be that, upon absorption at the peripheral tolane branches, population is transferred via a trans-stilbene geometry, where it must immediately couple to states localized in the next segment. Given the work presented above, out-of-plane trans-distortions might therefore also be expected to be playing a role in the energy funnelling mechanisms of this remarkable family of molecules.

\section{Acknowledgements}

The funding for this project was provided by the University of Birmingham 
[1] S. F. Swallen, R. Kopelman, J. S. Moore, C. Devadoss, Dendrimer photoantenna supermolecules: energetic funnels, exciton hopping and correlated excimer formation, J. Mol. Struct. 485 (1999) 585-597.

[2] M. A. Fox, Polymeric and supramolecular arrays for directional energy and electron-transport over macroscopic distances, Acc. Chem. Res. 25 (1992) 569-574.

[3] D. Nozaki, K. Yoshizawa, Molecular orbital engineering of singlemolecular light emission, Chem. Phys. Lett. 394 (2004) 194-197.

[4] Y. Tanizaki, H. Inoue, T. Hoshi, Shiraish.J, Localized and delocalized electronic transitions in diphenylacetylene, stilbene and diphenylbutadiene, Z. Phys. Chem. 74 (1971) 45-.

[5] K. Okuyama, T. Hasegawa, M. Ito, N. Mikami, Electronic-spectra of tolane in a supersonic free jet - large-amplitude torsional motion, J. Phys. Chem. 88 (1984) 1711-1716.

[6] Y. Hirata, T. Okada, N. Mataga, T. Nomoto, Picosecond time-resolved absorption-spectrum measurements of the higher excited singlet-state of diphenylacetylene in the solution phase, J. Phys. Chem. 96 (1992) 6559-6563.

[7] M. Gutmann, M. Gudipati, P. F. Schonzart, G. Hohlneicher, Electronicspectra of matrix-isolated tolan - site selective one-photon and 2-photon spectra, J. Phys. Chem. 96 (1992) 2433-2442.

[8] Z. Chernia, T. Livneh, I. Pri-Bar, J. E. Koresh, Mode assignment for linear phenyl acetylene sequence: phenylacetylene, di-phenylacetylene and 1,4-di(phenylethynyl)benzene, Vib. Spect. 25 (2001) 119-131.

[9] D. R. Borst, S. G. Chou, D. W. Pratt, Identification of the lightabsorbing states in tolane with potential relevance to self-similar phenylacetylene dendrimers, Chem. Phys. Lett. 343 (2001) 289-295.

[10] J. Saltiel, V. K. R. Kumar, Photophysics of diphenylacetylene: Light from the dark state, J. Phys. Chem. A 116 (2012) 10548-10558.

[11] T. Ishibashi, H. Hamaguchi, Structure and dynamics of s-2 and s-1 diphenylacetylene in solution studied by picosecond time-resolved cars spectroscopy, J. Phys. Chem. A 102 (1998) 2263-2269. 
[12] T. Suzuki, M. Nakamura, T. Isozaki, T. Ikoma, "dark" excited states of diphenylacetylene studied by nonresonant two-photon excitation opticalprobing photoacoustic spectroscopy, Int. J. Thermophys. 33 (2012) 2046-2054.

[13] E. K. L. Ho, T. Etienne, B. Lasorne, Vibronic properties of parapolyphenylene ethynylenes: TD-DFT insights, J. Chem. Phys. 146 (2017) 164303.

[14] V. D. Kleiman, J. S. Melinger, D. McMorrow, Ultrafast dynamics of electronic excitations in a light-harvesting phenylacetylene dendrimer, J. Phys. Chem. B 105 (2001) 5595-5598.

[15] C. Ferrante, U. Kensy, B. Dick, Does diphenylacetylene (tolan) fluoresce from its 2 nd excited singlet-state - semiempirical mo calculations and fluorescence quantum yield measurements, J. Phys. Chem. 97 (1993) $13457-13463$.

[16] M. Z. Zgierski, E. C. Lim, Nature of the 'dark' state in diphenylacetylene and related molecules: state switch from the linear pi pi* state to the bent pi sigma* state, Chem. Phys. Lett. 387 (2004) 352-355.

[17] Y. Amatatsu, M. Hosokawa, Theoretical study on the photochemical behavior of diphenylacetylene in the low-lying excited states, J. Phys. Chem. A 108 (2004) 10238-10244.

[18] M. Kramer, U. H. F. Bunz, A. Dreuw, Comprehensive look at the photochemistry of tolane, J. Phys. Chem. A 121 (2017) 946-953.

[19] H. Köppel, W. Domcke, L. S. Cederbaum, Multimode molecular dynamics beyond the born-oppenheimer approximation, Adv. Chem. Phys. 57 (1984) 59-246.

[20] G. A. Worth, H.-D. Meyer, H. Köppel, L. S. Cederbaum, I. Burghardt, Using the MCTDH wavepacket propagation method to describe multimode nonadiabatic dynamics, Int. Rev. Phys. Chem. 27 (2008) 569-606.

[21] C. Cattarius, G. A. Worth, H.-D. Meyer, L. S. Cederbaum, All mode dynamics at the conical intersection of an octa-atomic molecules: Multiconfiguration time-dependent Hartree (MCTDH) investigation on the butatriene cation, J. Chem. Phys. 115 (2001) 2088-2100. 
[22] A. Raab, G. A. Worth, H.-D. Meyer, L. S. Cederbaum, Molecular Dynamics Of Pyrazine After Excitation To The $\mathrm{S}_{2}$ Electronic State Using A Realistic 24-mode Model Hamiltonian., J. Chem. Phys. 110 (1999) 936-946.

[23] S. Mahapatra, G. A. Worth, H.-D. Meyer, L. S. Cederbaum, H. Köppel, The $\tilde{\mathrm{A}}^{2} \mathrm{E} / \tilde{\mathrm{B}}^{2} \mathrm{~B}_{2}$ photoelectron bands of allene beyond the linear coupling scheme: An ab initio dynamical study including all fifteen vibrational modes, J. Phys. Chem. A 105 (2001) 5567-5576.

[24] C. Robertson, G. A. Worth, Modelling the vibrationally mediated photodissociation of acetylene, PCCP 19 (2017) 29483-29497.

[25] H.-D. Meyer, U. Manthe, L. S. Cederbaum, The Multi-Configurational Time-Dependent Hartree Approach, Chem. Phys. Lett. 165 (1990) 7378.

[26] U. Manthe, H.-D. Meyer, L. S. Cederbaum, Wave-Packet Dynamics within the Multiconfiguration Hartree Framework: General Aspects and application to NOCl, J. Chem. Phys 97 (1992) 3199-3213.

[27] M. H. Beck, A. Jackle, G. A. Worth, H. D. Meyer, The multiconfiguration time-dependent hartree (mctdh) method: a highly efficient algorithm for propagating wavepackets, Phys. Rep. 324 (2000) 1-105.

[28] H.-J. Werner, P. J. Knowles, G. Knizia, F. R. Manby, M. Schütz, P. Celani, T. Korona, R. Lindh, A. Mitrushenkov, G. Rauhut, K. R. Shamasundar, T. B. Adler, R. D. Amos, A. Bernhardsson, A. Berning, D. L. Cooper, M. J. O. Deegan, A. J. Dobbyn, F. Eckert, E. Goll, C. Hampel, A. Hesselmann, G. Hetzer, T. Hrenar, G. Jansen, C. Köppl, Y. Liu, A. W. Lloyd, R. A. Mata, A. J. May, S. J. McNicholas, W. Meyer, M. E. Mura, A. Nicklass, D. P. O'Neill, P. Palmieri, D. Peng, K. Pflüger, R. Pitzer, M. Reiher, T. Shiozaki, H. Stoll, A. J. Stone, R. Tarroni, T. Thorsteinsson, M. Wang, Molpro, version 2012.1, a package of ab initio programs (2012).

[29] S. Grimme, M. Waletzke, A combination of kohn-sham density functional theory and multi-reference configuration interaction methods, J. Chem. Phys. 111 (1999) 5645-5655. 
[30] C. Adamo, D. Jacquemin, The calculations of excited-state properties with time-dependent density functional theory, Chem. Soc. Rev. 42 (2013) 845-856.

[31] M. J. Frisch, G. W. Trucks, H. B. Schlegel, G. E. Scuseria, M. A. Robb, J. R. Cheeseman, J. A. Montgomery, Jr., T. Vreven, K. N. Kudin, J. C. Burant, J. M. Millam, S. S. Iyengar, J. Tomasi, V. Barone, B. Mennucci, M. Cossi, G. Scalmani, N. Rega, G. A. Petersson, H. Nakatsuji, M. Hada, M. Ehara, K. Toyota, R. Fukuda, J. Hasegawa, M. Ishida, T. Nakajima, Y. Honda, O. Kitao, H. Nakai, M. Klene, X. Li, J. E. Knox, H. P. Hratchian, J. B. Cross, V. Bakken, C. Adamo, J. Jaramillo, R. Gomperts, R. E. Stratmann, O. Yazyev, A. J. Austin, R. Cammi, C. Pomelli, J. W. Ochterski, P. Y. Ayala, K. Morokuma, G. A. Voth, P. Salvador, J. J. Dannenberg, V. G. Zakrzewski, S. Dapprich, A. D. Daniels, M. C. Strain, O. Farkas, D. K. Malick, A. D. Rabuck, K. Raghavachari, J. B. Foresman, J. V. Ortiz, Q. Cui, A. G. Baboul, S. Clifford, J. Cioslowski, B. B. Stefanov, G. Liu, A. Liashenko, P. Piskorz, I. Komaromi, R. L. Martin, D. J. Fox, T. Keith, M. A. Al-Laham, C. Y. Peng, A. Nanayakkara, M. Challacombe, P. M. W. Gill, B. Johnson, W. Chen, M. W. Wong, C. Gonzalez, J. A. Pople, Gaussian 03, Revision C.02.

[32] J. L. Palma, E. Atas, L. Hardison, T. B. Marder, J. C. Collings, A. Beeby, J. S. Melinger, J. L. Krause, V. D. Kleiman, A. E. Roitberg, Electronic spectra of the nanostar dendrimer: Theory and experiment, J. Phys. Chem. C 114 (2010) 20702-20712.

[33] B. Schmidt, C. Sobotta, S. Malkmus, S. Laimgruber, M. Braun, W. Zinth, P. Gilch, Femtosecond fluorescence and absorption dynamics of an azobenzene with a strong push-pull substitution, J. Phys. Chem. A 108 (2004) 4399-4404.

[34] I. Hartl, P. Gilch, W. Zinth, Ultrafast redistribution of vibrational excitation of ch-stretching modes probed via anti-stokes raman scattering, Appl. Phys. B 71 (2000) 397-403. 